This is a post-peer-review, pre-copyedit version of an article published in Clinical Drug Investigation. The final authenticated version is available online at: https://doi.org/10.1007/s40261-020-00891-w. 


\section{Budget Impact Analysis of Dalbavancin in the Treatment of Acute Bacterial Skin and Skin Structure Infections in Three European Countries}

Andrea Marcellusi ${ }^{1,2}$, Chiara Bini ${ }^{1}$, Massimo Andreoni ${ }^{3}$, Loredana Sarmati ${ }^{3}$, Jaime Espin ${ }^{4}$, Juan P. Horcajada ${ }^{5}$, Thomas Czypionka ${ }^{6,7}$, Davide Andretta ${ }^{8}$, Paolo Sciattella ${ }^{1}$, Giampiero Favato ${ }^{2}$, Francesco S. Mennini ${ }^{1,2}$

${ }^{1}$ CEIS- Economic Evaluation and HTA (EEHTA), Faculty of Economics, University of Rome "Tor Vergata", Italy.

${ }^{2}$ Institute for Leadership and Management in Health - Kingston University London, London, UK.

${ }^{3}$ Clinical Infectious Diseases, Department of Systems Medicine, University of Rome "Tor Vergata", Rome, Italy.

${ }^{4}$ Andalusian School of Public Health, Granada, Spain.

${ }^{5}$ Department of Infectious Diseases Hospital del Mar, Institut Hospital del Mar d'Investigacions Mèdiques (IMIM), Barcelona, Spain.

${ }^{6}$ Institute for Advanced Studies, Vienna, Austria.

${ }^{7}$ London School of Economics, London, UK.

${ }^{8}$ Global HEOR Angelini Spa, Rome, Italy.

Corresponding author: Dr Andrea Marcellusi

Institute: Economic Evaluation and HTA (EEHTA) - Faculty of Economics

University: University of Rome "Tor Vergata", Rome, Italy.

Address: Via Columbia 2

Postal code: 00133 Rome - Italy

E-mail: andrea.marcellusi@uniroma2.it

\section{Short title:}

Budget Impact Analysis of Dalbavancin for ABSSSI in Italy, Spain and Austria 


\section{Abstract}

Background and Objective: Acute bacterial skin and skin structure infections have been defined by Food and Drug Administration (FDA) in 2013 to define a subset of complicated skin and skin structure infections commonly treated with parenteral antibiotic therapy. Inpatient treatment of ABSSSIs involves a significant economic burden on the health-care system. This study aimed to evaluate the economic impact on National Health System associated with the management of non-severe ABSSSIs treated in hospitals with innovative long-acting dalbavancin compared to standard antibiotic therapy in Italy, Spain and Austria.

Methods: A Budget Impact Analysis was developed to evaluate the direct costs associated with the management of ABSSSI from the national public health system perspective. The model considered the possibility to early discharge patients directly from the Emergency Department (ED), after one night in the hospital or after 2 or 3 night in the hospital. A scenario with Standard of Care was compared with dalbavancin scenario, where patients had the possibility of being early discharged. The epidemiological and cost parameters were extrapolated from national administrative databases and from a systematic literature review for each Country. The analysis was conducted in a 3-year time horizon. A one-way deterministic sensitivity analysis was conducted to examine the robustness of the results.

Results: The model estimated an average annual number of patients with non-severe ABSSSI in Italy, Spain and Austria equal to 5,396, 7,884 and 1,788 respectively. A total annual expenditure of about $€ 9.9$ million, $€ 13.5$ million and $€ 3.4$ million was estimated for treating the full set of ABSSSI patients in Italy, Spain and Austria respectively. Dalbavancin reduced the in-hospital length of stay in each Country. In the first year of its introduction, Dalbavancin significantly reduced the total economic burden in Italy and Spain $(-€ 352,252$ and $-€ 233,991)$ while it increased the total economic burden in Austria ( $€ 80,769,0.7 \%$ of the total expenditure for these patients); in the third year of its introduction, Dalbavancin reduced the total economic burden in each Country ( $€$ 1,1 million, $-€ 810,650,-€ 70,269$ respectively).

Conclusions: The introduction of dalbavancin in a new patients pathway to treat non-severe aBSSSI, could generate a significant reduction of hospitalized patients and the overall patient's length of stay in hospital 


\section{Key Points:}

- Dalbavancin reduces the in-hospital length of stay in each Country.

- The introduction of dalbavancin in a new patients pathway to treat non-severe aBSSSI could reduce the total economic burden in each Country.

\section{Introduction}

Skin and skin structure infection (SSSI) represents one of the most frequent infections treated in a hospital setting $[1,2]$. Skin and soft tissue infection are generally defined as complicated (CSSTI) when they require inpatient management or surgical procedures or they are associated with comorbidities, such as diabetes or systemic immunosuppression, that can complicate a patient's response to treatment [1].

In 2013, the US Food and Drug Administration (FDA) introduced the term "acute bacterial skin and skin structure infections" (ABSSSIs) to define a subset of complicated SSTIs commonly treated with parenteral antibiotic therapy [3]. Based on the FDA guidelines, ABSSSIs include cellulitis, erysipelas, wound infections, and major cutaneous abscesses with a minimum lesion surface area of $75 \mathrm{~cm}^{2}$ [3].

Many pre-existing chronic conditions, such as obesity, lymphedema, venous insufficiency, and prior trauma to the area, have been associated with an increased risk of developing ABSSSIs $[4,5]$. Common bacterial pathogens causing ABSSSI are Streptococcus pyogenes and Staphylococcus aureus, including methicillin-resistant strains (MRSA) [3]. Less commonly identified bacteria include other Streptococcus species, Enterococcus faecalis, and gram-negative bacteria [3]. Staphylococcus aureus is the most common cause of ABSSSIs in all regions of North America, Latin America and Europe [6].

The European Union (EU) and European Economic Area (EEA) population-weighted mean MRSA percentage decreased significantly from $19.6 \%$ in 2014 to $16.9 \%$ in 2017; 
nevertheless, MRSA remains an important pathogen in the EU/EEA since the levels of MRSA are still high in several countries [7].

The highest percentages of invasive isolates with methicillin resistance (MRSA) have been observed in Spain, Portugal, Italy, Greece, Bulgaria, Croatia and Slovakia, with ranges from 25\% to 50\%; followed by France, Ireland, the Czech Republic, Poland, Hungary and Romania, with ranges from $10 \%$ to $25 \%$; and the UK, Belgium, Germany, Austria, Slovenia, Lithuania and Latvia, with ranges from $5 \%$ to $10 \%$ [7].

The most serious ABSSSIs that require hospitalization for parenteral antibiotic therapy are diagnosed on the basis of the virulence and resistance patterns of the causative pathogens and host factors, which can potentially increase the severity of the infection $[1,8-11]$. If misdiagnosed or not managed appropriately, these acute infections can lead to complications that may have a devastating impact on the quality of life of patients, and they may increase the risk of mortality [12]. According to recent guidelines [4], ABSSSIs are classified based on clinical severity as mild, moderate or severe infections. Severe ABSSSIs are those that do not respond to empirical antibiotic treatment and/or surgical drainage and are generally associated with signs of systemic infection (fever $>38^{\circ} \mathrm{C}$, elevated white blood cells). ABSSSIs in immunocompromised patients are also considered severe. Mild skin infections are usually resolved with incision and drainage, while moderate ABSSSIs could benefit from incision and drainage but commonly require a short course of antibiotic therapy, thus often resulting in a short duration of hospitalization. For patients with severe infections, clinical reassessment is usually performed 72 hours after hospitalization (discharge on day 4 or prolonged observation until day 8), while the treatment duration is indicated to be 7-14 days (discharge on day 8 or prolonged observation, corresponding to hospitalization for more than 8 days). Patients with moderate (non-severe) infections are generally hospitalized for $\leq 2-3$ days.

The 2014 Practice Guidelines for the Diagnosis and Management of Skin and Soft Tissue Infections released by the Infectious Diseases Society of America (IDSA) identified two additional categories of ABSSSIs: non-purulent (cellulitis and erysipelas) and purulent (abscesses, carbuncles, and furuncles). The guidelines also recommended an algorithm for the management of each type of infection based on differences in microbiology and severity of illness [4]. These infections are more likely to be caused by Staphylococcus aureus, including MRSA, which has been identified as the aetiological cause of infection in up to $60 \%$ 
of the positive cultures from ABSSSIs from accident and emergency departments (A\&EDs) [13].

Based on this evidence, the US FDA is looking at early clinical response, defined as the cessation of spread of the primary lesion and resolution of fever within 48-72 hours after enrolment, as the main endpoint for the approval of new antibiotic therapies indicated for the treatment of ABSSSIs. New approved agents have been designed to decrease the rate of hospitalization and the length of stay (LOS) (with both intravenous and oral formulations available for ease of transition at discharge), while improving safety/tolerability and increasing the spectrum of activity to act against increasingly resistant pathogens [14]. There may be a potential benefit of cost-prevention by decreasing the LoS and reducing the need for hospital admission through the administration of long-acting lipoglycopeptides in either inpatient or outpatient settings, although supporting data are limited [14].

Dalbavancin is a semisynthetic lipoglycopeptide that is active against several multidrug-resistant gram-positive bacteria, including MRSA [15]. The main advantage of dalbavancin is its long half-life (up to 14 days), which allows once-weekly administration and an early hospital discharge [15]. In the DISCOVER-1 and DISCOVER-2 randomized clinical trials (RCTs), dalbavancin was not inferior to twice-daily intravenous vancomycin followed by oral linezolid for the treatment of ABSSSIs. The most common adverse events (AEs) reported were mild gastrointestinal side effects that rarely led to the discontinuation of the treatment [16].

A previous study aimed to evaluate the direct costs associated with the management of severe ABSSSI patients from a national health-care provider's perspective in Italy, Romania, and Spain. The administration of dalbavancin resulted in a mean reduction in hospital stay of 3.3 days per ABSSSI patient, with no significant incremental costs from a National Health System perspective [17]. This paper concluded that the use of dalbavancin could allow early discharge ABSSSI management, potentially significantly reducing patients' exposure to additional risks associated with prolonged hospitalization, with no incremental cost for the National healthcare providers. What happens to the non-severe ABSSSIs? Could they have advantages due to the long-acting bactericidal activity of and unique dosing schedule for dalbavancin?

The objective of this research is to evaluate the economic impact on National Health System associated with the management of non-severe ABSSSIs (moderate ABSSSIs, 
according to IDSA definition) treated in hospitals with innovative long-acting dalbavancin respect to standard antibiotic therapy in Italy, Spain and Austria.

\section{Methods}

A budget impact analysis (BIA) was developed to estimate the direct costs associated with the management of ABSSSIs from the public National Health System perspective. The methodology followed the guidelines recommended by the International Society of Pharmacoeconomics and Outcome Research (ISPOR) Principles of Good Practice [21]. Similar to a previously published paper [17], literature data and clinical experts' opinions were used as inputs in the model when primary data were not available [21].

A decision-analytic model was developed on the basis of the current clinical practices in three European countries with the aim of simulating the hospital management of nonsevere ABSSSI patients receiving empiric treatment with antibiotics (Figure 1).

The analysis was conducted over a 3-year time period considering the standard guidelines suggested for a BIA [21].

The choice of the countries was based on the percentages of invasive isolates with methicillin resistance (MRSA) registered in each country. We considered two countries with high levels of MRSA (Italy and Spain) and one country with a lower percentage (Austria) [7].

Most of the 28 countries in the European Union have a publicly funded national system that provides universal access to healthcare. However, the relevance of MRSA widely varies around the EU28 (mean value of $16.9 \%$ in 2017) [7]. Based on the relevance of this epidemiological impact, the simulation included the two EU countries with the higher percentage of MRSA (Italy and Spain, $25-50 \%$ in 2017) and the country with the lowest level of MRSA (Austria, $5-10 \%$ ) [7].

\subsection{Eligible population}

Eligible patients were identified using the national administrative databases of each country (Appendix A). The algorithm included all acute inpatient admissions. The data collection period per country was selected based on the available data by country: Italy and 
Spain, January 1st, 2006, to December 31st, 2014; and Austria, January 1st, 2008, to December 31st, 2014.

\subsection{Intervention comparison and model structure}

The decision tree model reported in Figure 1 shows that all ABSSSI patients can undergo the following:

a) early discharge (directly from the emergency department - ED),

b) discharge after one night in the hospital,

c) discharge after 2 or 3 night in the hospital (first probabilistic node).

The patients initially receive empirical antibiotic treatment to cover gram-positive infections and remain in the hospital for an observational period of a few hours (a), for 24 hours (b) or for 2 or 3 nights (c). The model considers that the patients could receive either a prescription for oral antibiotics before discharge (current intervention or standard of care $\mathrm{SoC}$ ) or the administration of dalbavancin (new intervention). The choice of antibiotic combination therapy was based on the IDSA guidelines for the treatment of non-severe ABSSIs [18].

After receiving antibiotic therapy and discharge, the patients could have a successful clinical response (no additional hospitalization due to aBSSSI) or a poor clinical response (leading to a new admission to the hospital - second probabilistic node). Patients with a poor clinical response could present clinical worsening or a severe clinical worsening (third probabilistic node). The transition probabilities change according to the treatment options (SoC or dalbavancin) administered on day 0.

\section{Figure 1: Decision tree}

\footnotetext{
ABSSSIs: Acute Bacterial Skin and Skin Structure Infections

Discharged: early discharge (directly from the emergency department - ED)

Hospitalized 0-1: hospitalization for 0-1 day (1 night)

Hospitalized 2-3: hospitalization for 2-3 days (2-3 nights)

Successful clinical response: no additional hospitalization due to aBSSSI

Poor clinical response: leading to a new admission to the hospital

Non severe clinical worsening: re-hospitalization with a length of stay (LoS) $<4$ days

Severe clinical worsening: re-hospitalization with a length of stay (LoS) $\geq 4$ days
} 


\subsection{Non-monetary inputs to the model}

The input values of the probabilistic nodes are reported in Table 1.

For the SoC scenario, the proportion of non-severe ABSSSI patients in the two starting groups (0-1 days or 2-3 days in hospital) was obtained from the real-world databases of each country (details are provided in the appendix).

In the dalbavancin administration scenario, the starting distribution could change accordingly to the following: 1) the number of patients treated with dalbavancin, 2) the percentage of patients who could be discharged earlier than SoC-treated patients and 3) the reduced rate of re-hospitalization.

Regarding the number of patients treated with dalbavancin, the model assumes that an increasing number of patients during the 3-year follow-up will be treated with the new antibiotic depending on the severity of the disease and the observational period required in the SoC scenario (Table 1).

The ability to reduce the number of hospitalization days attributed to the administration of dalbavancin was based on experts' opinions and was supported by their current clinical practices. Replacing the current SoC (empirical antibiotic treatment) with the early administration of dalbavancin could potentially lead to a significant reduction in the mean hospitalization time associated with non-severe ABSSSI:

- (a) Day 0: Dalbavancin administered in the ED could reduce the number of patients who remain in the hospital for 1 or 2-3 nights (assumed equal to 90\% for each treated patient in the base-case scenario - Table 1);

- (b) Day 1: Dalbavancin administered after one day of observation could reduce the number of patients who remain in the hospital for a second or third day);

- (c) Day 2-3: Some patients remain in the hospital even if they were previously treated with dalbavancin on days 0 or 1 . The base case analysis assumes that patients who stay in the hospital for 2-3 days in the SoC scenario, when treated with dalbavancin, are discharged directly from the ED in $15 \%$ of the cases, while 
the rest of the treated patients are discharged after one day (Table 1). This percentage increases in years two and three (30 and 50\%, respectively), assuming increasing confidence in the new drug by physicians.

The model conservatively assumes that the risk of clinical worsening remains the same if patients are treated with either SoC or dalbavancin. 
Table 1: Transition probabilities: SoC (real-world data) vs. dalbavancin (expert opinion)

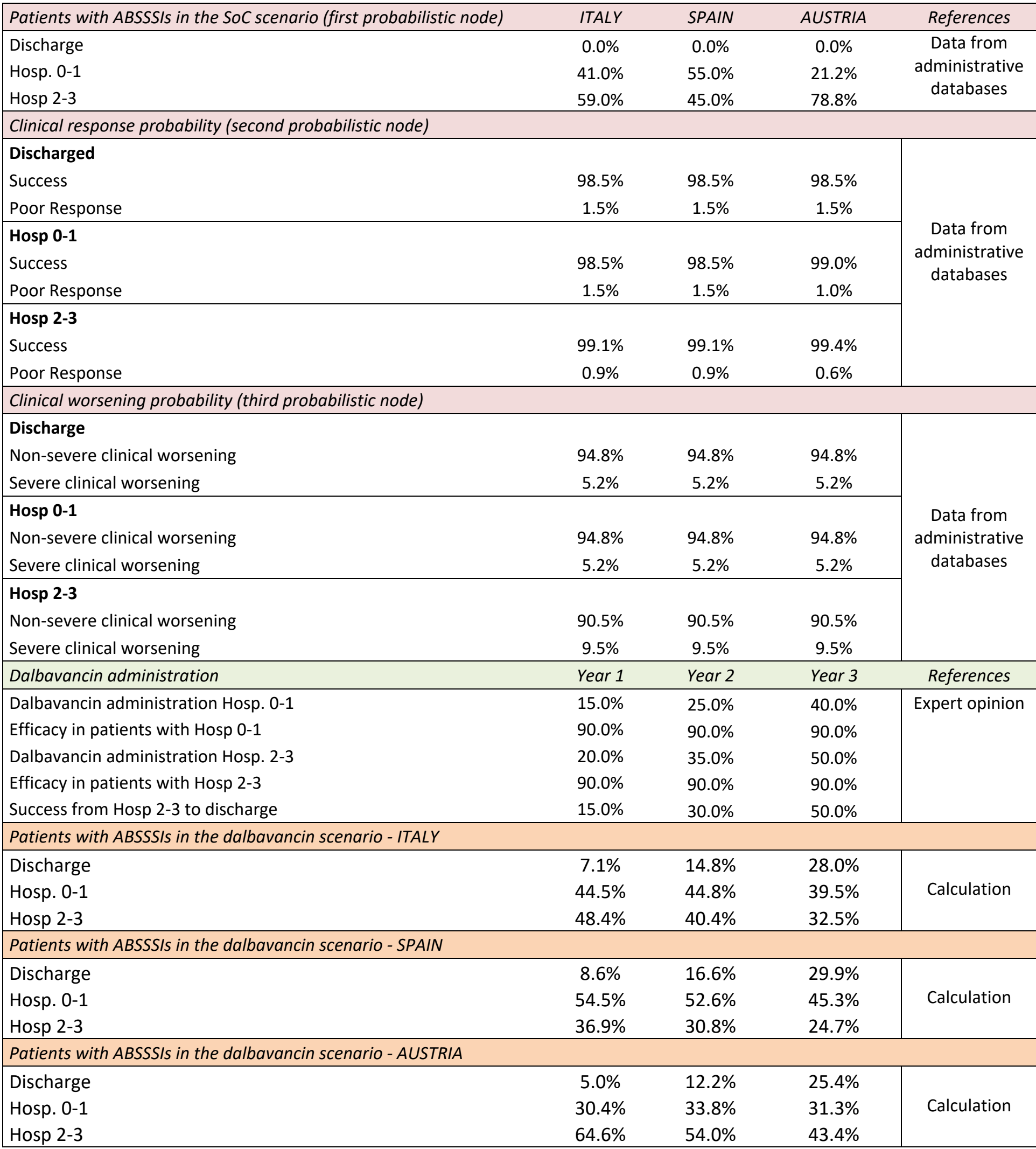

SoC: Standard of Care

ABSSSIs: Acute Bacterial Skin and Skin Structure Infections

Hosp 0-1: hospitalization for 0-1 day (1 night)

Hosp 2-3: hospitalization for 2-3 days (2-3 nights) 


\subsection{Cost inputs in the model}

Tariffs and direct costs associated with each hospitalization type and length of stay were used as inputs to inform the model (Table 2). For Italy, the costs were derived from the Italian National Tariffs associated with each specific diagnosis-related group (DRG), as reported in the epidemiological and risk analysis (Appendix). For patients with clinical worsening, the estimated costs of re-hospitalization were considered, assuming a LoS $<4$ days for non-severe worsening and a LoS $\geq 4$ days for severe worsening.

For Spain, the hospitalization costs were derived from the official Spanish database (http://pestadistico.inteligenciadegestion.msssi.es/publicosns), which includes tariffs for minor patients (LOS $=0-1$ days) or moderate patients (LOS $=2-3$ days). Due to a lack of data, the average hospitalization costs due to clinical worsening were increased by the same percentages used in the Italian scenario.

For Austria, the required data were not available publicly. Moreover, ABSSSIs are not clearly identifiable in the DRG system but are mostly a component of a wider DRG category. Therefore, a data request was made to cross-reference DRG and ICD information on different LoSs (for details see appendix). As only part of the hospital costs are paid by the DRG tariff, the latter does not reflect the economic costs of the treatment [19]. To adjust for this, we used information on total inpatient costs from the Austrian hospital accounting system, also requested from the $\mathrm{MoH}$. However, the perspective of this study is that of the national health system; therefore, we deducted private payments for hospital inpatient stays, as these payments mostly cover additional amenities that incur costs not covered by the public system. The remaining inpatient costs were divided by the total DRG points to obtain cost/DRG, which can be applied to the DRG points of relevant stays.

In the model, the dalbavancin drug cost was estimated considering that patients are treated with a single infusion of $1500 \mathrm{mg}$ at day 0 (Table 2). 
Table 2: Cost inputs for each country included in the analysis (year 2018)

\begin{tabular}{|c|c|c|c|c|}
\hline Cost parameters & ITALY & SPAIN & AUSTRIA & References \\
\hline Admitted to ED & $€ 0.0$ & $€ 0.0$ & $€ 0.0$ & \multirow{9}{*}{$\begin{array}{c}\text { Data from } \\
\text { administrative } \\
\text { databases } \\
\text { (Appendix A) }\end{array}$} \\
\hline Admitted to ED with clinical worsening & $€ 1,089.1$ & $€ 1,238.0$ & $€ 1,461.7$ & \\
\hline Admitted to ED with severe clinical worsening & $€ 3,163.0$ & $€ 3,595.5$ & $€ 3,806.9$ & \\
\hline Hosp 0-1 & $€ 490.0$ & $€ 557.0$ & $€ 1,228.0$ & \\
\hline Hosp 0-1 with clinical worsening & $€ 1,089.1$ & $€ 1,238.0$ & $€ 2,535.3$ & \\
\hline Hosp 0-1 with severe clinical worsening & $€ 3,163.0$ & $€ 3,595.5$ & $€ 4,880.6$ & \\
\hline Hosp 2-3 & $€ 2,735.3$ & $€ 3,073.8$ & $€ 2,085.4$ & \\
\hline Hosp 2-3 with clinical worsening & $€ 2,226.1$ & $€ 2,501.6$ & $€ 3,392.7$ & \\
\hline Hosp 2-3 with severe clinical worsening & $€ 2,748.7$ & $€ 3,088.9$ & $€ 5,737.9$ & \\
\hline Dalbavancin & $€ 1,161.0$ & $€ 1,290.0$ & $€ 1,200.0^{*}$ & [17] \\
\hline
\end{tabular}

* Estimated cost.

ED: Emergency Department

Hosp 0-1: hospitalization for 0-1 day (1 night)

Hosp 2-3: hospitalization for 2-3 days (2-3 nights)

\subsection{Sensitivity analysis}

One-way deterministic sensitivity analysis (DSA) was performed to evaluate the impact of uncertainty of input parameters on the results. Such analysis consists of changing one input parameter at a time, according to the minimum and maximum values found in the literature or assumed by the authors. In this specific case, the minimum and maximum values were defined assuming $\pm 10 \%$ variability for each parameter.

\section{RESULTS}

Figure 2 shows the average annual number of patients affected by non-severe ABSSSIs in each country included in the analysis. The model identified 5,396 non-severe ABSSSIs patients in Italy; $41 \%(2,212)$ of these patients were hospitalized with a LoS of $0-1$ days, while $59 \%(3,183)$ were hospitalized with a LoS of 2-3 days. In Spain, 7,884 non-severe ABSSSIs patients were identified; $55 \%(4,337)$ of these patients were hospitalized with a LoS of 0-1 days, and $45 \%(3,547)$ were hospitalized with a LoS of 2-3 days. In Austria, 1,788 non-severe 
ABSSSIs patients were identified; $21.2 \%$ (378) had a LoS of $0-1$ days and $78.8 \%$ were hospitalized with a LoS of 2-3 days.

Figure 2: Average annual number of patients affected by non-severe ABSSSIs hospitalized in Italy (2006-2014), Spain (2006-2014), and Austria (2008-2014).

ABSSSIs: Acute Bacterial Skin and Skin Structure Infections

LoS: Length of stay

0-1 day: hospitalization for $0-1$ day (1 night)

2-3 days: hospitalization for 2-3 days (2-3 nights)

After the first year of observation, dalbavancin treatment reduced the in-hospital LoS by approximately 230 days, 207.4 days and 261.8 days per 1,000 hospitalised ABSSSI patients in Italy, Spain and Austria, respectively. By the third year of observation, these reductions increased to $-677.1,-601.8$ and -782.6 days per 1,000 hospitalized ABSSSI patients in Italy, Spain and Austria, respectively (Figure 2).

From an economic perspective, the model estimated a total annual expenditure of $€$ 9.9 million, $€ 13.5$ million and $€ 3.4$ million for the treatment of all hospitalized non-severe ABSSSI patients in Italy, Spain and Austria, respectively (Table 4). In Italy, the early use of dalbavancin decreased the total annual treatment costs by $€ 352,252$ in year 1 and $€ 1.1$ million in year 3. Similar annual savings, ranging from $€ 233.991$ (year 1 ) to $€ 810,650$ (year 3), were realized in Spain. In Austria, dalbavancin appeared to be associated with a moderate total annual increase of $€ 80.769$ in the first year of observation, but this was offset by an estimated cost savings of $€ 70.269$ in the last year of the simulation (Table 4).

Figure 3: In-hospital length of stay (days per 1,000 hospitalized ABSSSI patients) in the SoC and dalbavancin scenarios

SoC: Standard of Care 
Table 4: Annual costs for the SoC and dalbavancin scenarios and budget impact results

\begin{tabular}{|c|c|c|c|c|c|c|c|c|c|}
\hline & \multicolumn{3}{|c|}{ SoC } & \multicolumn{3}{|c|}{ Dalbavancin } & \multicolumn{3}{|c|}{ Difference (dalbavancin - SoC) } \\
\hline & Italy & Spain & Austria & Italy & Spain & Austria & Italy & Spain & Austria \\
\hline & \multicolumn{9}{|c|}{ Year 1} \\
\hline Discharged & $€ 0.0$ & $€ 0.0$ & $€ 0.0$ & $€ 453,544.4$ & $€ 893,009.0$ & $€ 109,152.2$ & $€ 453,544.4$ & $€ 893,009.0$ & $€ 109,152.2$ \\
\hline Hosp 0-1 & $€ 1,124,354.1$ & $€ 2,505,808.5$ & $€ 474,578.7$ & $€ 1,824,126.6$ & $€ 3,265,132.4$ & $€ 946,686.8$ & $€ 699,772.5$ & $€ 759,323.9$ & $€ 472,108.1$ \\
\hline Hosp 2-3 & $€ 8,774,934.2$ & $€ 10,988,014.6$ & $€ 2,968,488.6$ & $€ 7,269,365.6$ & $€ 9,101,691.1$ & $€ 2,467,997.3$ & $-€ 1,505,568.6$ & $-€ 1,886,323.5$ & $-€ 500,491.4$ \\
\hline \multirow[t]{2}{*}{ Total } & $€ 9,899,288.2$ & $€ 13,493,823.1$ & $€ 3,443,067.3$ & $€ 9,547,036.6$ & $€ 13,259,832.5$ & $€ 3,523,836.2$ & $-€ 352,251.6$ & $-€ 233,990.6$ & $€ 80,768.9$ \\
\hline & \multicolumn{9}{|c|}{ Year 2} \\
\hline Discharged & $€ 0.0$ & $€ 0.0$ & $€ 0.0$ & $€ 941,734.6$ & $€ 1,718,501.5$ & $€ 267,362.5$ & $€ 941,734.6$ & $€ 1,718,501.5$ & $€ 267,362.5$ \\
\hline Hosp 0-1 & $€ 1,124,354.1$ & $€ 2,505,808.5$ & $€ 474,578.7$ & $€ 2,107,310.3$ & $€ 3,542,772.7$ & $€ 1,142,059.0$ & $€ 982,956.2$ & $€ 1,036,964.1$ & $€ 667,480.3$ \\
\hline Hosp 2-3 & $€ 8,774,934.2$ & $€ 10,988,014.6$ & $€ 2,968,488.6$ & $€ 6,140,189.1$ & $€ 7,686,948.5$ & $€ 2,092,628.7$ & $-€ 2,634,745.0$ & $-€ 3,301,066.1$ & $-€ 875,859.9$ \\
\hline \multirow[t]{2}{*}{ Total } & $€ 9,899,288.2$ & $€ 13,493,823.1$ & $€ 3,443,067.3$ & $€ 9,189,234.1$ & $€ 12,948,222.7$ & $€ 3,502,050.2$ & $-€ 710,054.2$ & $-€ 545,600.4$ & $€ 58,982.9$ \\
\hline & \multicolumn{9}{|c|}{ Year 3} \\
\hline Discharged & $€ 0.0$ & $€ 0.0$ & $€ 0.0$ & $€ 1,783,827.0$ & $€ 3,092,740.1$ & $€ 555,166.5$ & $€ 1,783,827.0$ & $€ 3,092,740.1$ & $€ 555,166.5$ \\
\hline Hosp 0-1 & $€ 1,124,354.1$ & $€ 2,505,808.5$ & $€ 474,578.7$ & $€ 2,017,961.2$ & $€ 3,318,227.4$ & $€ 1,100,372.1$ & $€ 893,607.1$ & $€ 812,418.8$ & $€ 625,793.4$ \\
\hline Hosp 2-3 & $€ 8,774,934.2$ & $€ 10,988,014.6$ & $€ 2,968,488.6$ & $€ 5,011,012.7$ & $€ 6,272,205.9$ & $€ 1,717,260.2$ & $-€ 3,763,921.5$ & $-€ 4,715,808.7$ & $-€ 1,251,228.5$ \\
\hline \multirow[t]{2}{*}{ Total } & $€ 9,899,288.2$ & $€ 13,493,823.1$ & $€ 3,443,067.3$ & $€ 8,812,800.8$ & $€ 12,683,173.3$ & $€ 3,372,798.8$ & $-€ 1,086,487.5$ & $-€ 810,649.8$ & $-€ 70,268.5$ \\
\hline & & & & & $\begin{array}{l}\text { Overall period } \\
\text { (cumulative } \\
\text { budget impact) }\end{array}$ & & & & \\
\hline Total & $€ 29,697,864.7$ & $€ 40,481,469.4$ & $€ 10,329,202.0$ & $€ 27,549,071.5$ & $€ 38,891,228.6$ & $€ 10,398,685.2$ & $-€ 2,148,793.3$ & $-€ 1,590,240.8$ & $€ 69,483.2$ \\
\hline
\end{tabular}

\section{SoC: Standard of Care}

Hosp 0-1: hospitalization for 0-1 day (1 night)

Hosp 2-3: hospitalization for 2-3 days (2-3 nights) 
Figures $4 a, 4 b$ e $4 c$, shows the results of the deterministic sensitivity analyses conducted for each country; the variable that mainly affected the cumulative budget impact of each country was the second probabilistic node for patients who stay in the hospital for 2-3 days, both for SoC and dalbavancin scenario. In all the simulated scenario, dalbavancin represent a cost-saving option for Italy and Spain with a cumulative cost reduction between $€ 4.3-€ 0.7$ and $€ 4.3-€ 0.3$ respectively (Figure 4A and $4 C$ ). A similar effect was reported for Austria, where the variability over the main considered parameters estimates an incremental cost between - $€ 0.4$ million if we increase the probability of successful clinical response of dalbavancin and $+€ 1.1$ million if we decrease the Dalbavancin effect in the second node (Figura 4 C).

Figure 4: Results of the one-way deterministic sensitivity analyses for each country-Tornado plots

Figure 4A: cumulative budget impacts for Italy

Figure 4B: cumulative budget impacts for Spain

Figure 4C: cumulative budget impacts for Austria

Hosp 0-1: hospitalization for 0-1 day (1 night)

Hosp 2-3: hospitalization for 2-3 days (2-3 nights)

ABSSSIs: Acute Bacterial Skin and Skin Structure Infections

SoC: Standard of Care 


\section{DISCUSSION}

We identified 15,068 non-severe ABSSSI patients per year hospitalized in Italy, Spain and Austria through the administrative database of each country. The model assumed that the early administration of dalbavancin could accelerate the discharge of non-severe ABSSSI patients who currently spending 2 or 3 days in hospital settings. Patients could be discharged directly from the A\&ED or their hospital LOS could be reduced. This new management approach could increase the quality of life of ABSSSI patients, who could be monitored at home, and reduce the overall costs from the NHS perspective by reducing the direct costs related to hospital management.

The early use of dalbavancin reduced the hospitalization costs in the major part of the scenarios considered for each country; in Italy and Spain, these reductions generated a total expenditure savings for each year of the analysis. In Austria, the estimated cost reductions related to hospitalizations with a length of stay of 2-3 days generated savings only in the third year of the analysis.

The outcomes of the model suggest that increasing the early use of dalbavancin could generate savings in the overall direct costs of management of non-severe ABSSSI patients from a NHS perspective.

For ABSSSIs due to MRSA, the incremental length of hospitalization is the key cost driver [20]. New long-acting antibiotics represent a potential opportunity for early discharge not only for severe patients [17] but also for non-severe patients. This approach could modify the management of these infections with a considerable reduction in hospitalization costs $[17,21,22]$. Our findings were confirmed by a recent observational retrospective study in 29 hospitals in Spain; 15/69 (21.7\%) patients were treated with dalbavancin for ABSSSIs, Staphylococcus aureus was identified as a major pathogen (11/15 patients), and $12 / 15$ (80.0\%) patients had a favourable clinical outcome (resolution of signs and symptoms related to bacterial infection) [23]. The use of dalbavancin was associated with an overall cost reduction and an estimated LoS reduction of 1160 days in their one-year analysis [23].

As in most economic models, this study has some limitations. First, due to the lack of countryspecific data, some transition probabilities and cost inputs were assumed to be the same as those estimated for the Italian scenario. These assumptions, however, were validated and discussed with key opinion leaders who agreed with the choice of inputs.

Second, consulting a panel of experts was the only way to identify the advantages associated with dalbavancin treatment for non-severe ABSSSIs patients. For example, the increase in the early 
discharge rate of patients over the time period of the analysis was based on scenarios designated by the panel of clinical experts. Third, the probabilities of discharge with a successful clinical response, unlike the probabilities of discharge with a poor clinical response, for a LoS of 0-1 day were assumed to be the same as the probabilities estimated for a LoS of 2-3 days. The same assumption was made for the corresponding direct costs.

Finally, the costs used were proxies derived from the DRG payments received. This might be one of the reasons why the use of dalbavancin in Austria was only cost-effective in the third year. As seen in Table 2, 2- to 3-day stays were approximately 5.5 times more expensive as 0- to 1-day stays in both Italy and Spain, but they were only 1.7 times more expensive as those in Austria. Therefore, despite the great potential to reduce long stays, especially in Austria, the cost-reducing effect is decreased. Adapting DRG weights similar to Italy and Spain would reflect the costs better, and the use of dalbavancin in Austria would probably then be associated with cost-savings in all years.

The model was designed under a conservative assumption that the probabilities related to clinical response and clinical worsening were the same between the current standard of care and the dalbavancin scenarios.

\section{CONCLUSION}

The high economic burden of non-severe ABSSSIs is dependent on the length of stay (LOS) in the hospital $[1,23-25]$. The characteristics of long-acting antibiotics could represent an opportunity for the targeted management of ABSSSIs.

The decision-analytic model developed on the basis of the current clinical practices in three European countries suggested that increasing the early use of dalbavancin could significantly reduce both hospitalization rates and lengths of stay in non-severe ABSSSI patients.

This new management approach could increase the quality of life of treated patients, who could be monitored at home, reducing the direct costs related to hospital management from a NHS perspective. 


\section{COMPLIANCE WITH ETHICAL STANDARDS FUNDING}

No external funding was used in the preparation of this manuscript.

\section{CONFLICT OF INTEREST}

L Sarmati, Thomas Czypionka , J Epsin, JP Horcajada, G Favato and M Andreoni have received consulting fees from Angelini SpA. D Andretta is employees of Angelini SpA. This study was supported by an unconditional grant from Angelini SpA. The authors confirm that the paper is an accurate representation of the study results. The authors have no other relevant affiliations or financial involvement with any organization or entity with a financial interest in or financial conflict with the subject matter or materials discussed in the manuscript apart from those disclosed. 


\section{References}

1. Pollack, C.V., Jr., et al., Acute bacterial skin and skin structure infections (ABSSSI): practice guidelines for management and care transitions in the emergency department and hospital. J Emerg Med, 2015. 48(4): p. 508-19.

2. Pfuntner, A., L.M. Wier, and C. Stocks, Most Frequent Conditions in U.S. Hospitals, 2011: Statistical Brief \#162, in Healthcare Cost and Utilization Project (HCUP) Statistical Briefs. 2006: Rockville (MD).

3. The United States Food and Drug Administration. Guidance for Industry Acute Bacterial Skin and Skin Structure Infections: Developing Drugs for Treatment. 2013; Available from: https://www.fda.gov/media/71052/download.

4. Stevens, D.L., et al., Practice guidelines for the diagnosis and management of skin and soft tissue infections: 2014 update by the Infectious Diseases Society of America. Clin Infect Dis, 2014. 59(2): p. e10-52.

5. Poulakou, G., E. Giannitsioti, and S. Tsiodras, What is new in the management of skin and soft tissue infections in 2016? Curr Opin Infect Dis, 2017. 30(2): p. 158-171.

6. Moet, G.J., et al., Contemporary causes of skin and soft tissue infections in North America, Latin America, and Europe: report from the SENTRY Antimicrobial Surveillance Program (1998-2004). Diagn Microbiol Infect Dis, 2007. 57(1): p. 7-13.

7. European Antimicrobial Resistance Surveillance Network (EARS-Net). Annual report 2017. Available from: https://ecdc.europa.eu/sites/portal/files/documents/EARS-Net-report2017-update-jan-2019.pdf.

8. Moran, G.J., et al., Acute bacterial skin infections: developments since the 2005 Infectious Diseases Society of America (IDSA) guidelines. J Emerg Med, 2013. 44(6): p. e397-412.

9. Falcone, M., et al., Acute bacterial skin and skin structure infections in internal medicine wards: old and new drugs. Intern Emerg Med, 2016. 11(5): p. 637-48.

10. CREST. Guidelines on the management of cellulitis in adults. 2005 [cited 201919 June]; Available from: http://www.acutemed.co.uk/docs/Cellulitis\%20guidelines,\%20CREST,\%2005.pdf.

11. Amin, A.N., et al., Hospitalist perspective on the treatment of skin and soft tissue infections. Mayo Clin Proc, 2014. 89(10): p. 1436-51.

12. Pulido-Cejudo, A., et al., Management of acute bacterial skin and skin structure infections with a focus on patients at high risk of treatment failure. Ther Adv Infect Dis, 2017. 4(5): p. 143-161.

13. Moran, G.J., et al., Methicillin-resistant S. aureus infections among patients in the emergency department. N Engl J Med, 2006. 355(7): p. 666-74.

14. Jaffa, R.K., et al., Novel developments in the treatment of acute bacterial skin and skin structure infections. Expert Opin Pharmacother, 2019: p. 1-10.

15. Bassetti, M., et al., The role of dalbavancin in skin and soft tissue infections. Curr Opin Infect Dis, 2018. 31(2): p. 141-147.

16. Boucher, H.W., et al., Once-weekly dalbavancin versus daily conventional therapy for skin infection. N Engl J Med, 2014. 370(23): p. 2169-79.

17. Marcellusi, A., et al., Economic evaluation of the treatment of Acute Bacterial Skin and Skin Structure Infections (ABSSSIs) from the national payer perspective: introduction of a new treatment to the patient journey. A simulation of three European countries. Expert Rev Pharmacoecon Outcomes Res, 2019: p. 1-19.

18. Galgiani, J.N., et al., 2016 Infectious Diseases Society of America (IDSA) clinical practice guideline for the treatment of coccidioidomycosis. Clin. Infect. Dis., 2016. 63(6): p. e112-46. 
19. Czypionka, T., et al., Efficiency, ownership, and financing of hospitals: the case of Austria. Health Care Manag Sci, 2014. 17(4): p. 331-47.

20. Nathwani, D., Impact of methicillin-resistant Staphylococcus aureus infections on key health economic outcomes: does reducing the length of hospital stay matter? J Antimicrob Chemother, 2003. 51 Suppl 2: p. ii37-44.

21. Tarricone, R., et al., How complicated skin and soft tissue infections are treated in Italy: economic evaluation of inpatient intravenous antibiotic treatment in seven hospitals. J Med Econ, 2008. 11(2): p. 265-79.

22. Belisari, A. and L.G. Mantovani. Aspetti economici della gestione delle infezioni batteriche acute di cute e struttura cutanea. 2016; Volume 5, Number 4:[Available from: https://www.ijph.it/pdf/2016-v5-n4.pdf.

23. Bouza, E., et al., Dalbavancin in the treatment of different gram-positive infections: a reallife experience. Int J Antimicrob Agents, 2018. 51(4): p. 571-577. 


\section{Appendix A.}

\section{Algorithm to identify ABSSSI patients in Italy (non-severe)}

The following algorithm was implemented for all acute inpatient admissions with discharge dates between January $1^{\text {st }}, 2006$, and December $31^{\text {th }}, 2014$, in the Italian Hospital Information System (HIS).

To define non-severe patients requiring observation over 12 hours, one of the following inclusion criteria had to have been met:

All acute inpatient admissions with discharge

AND a primary diagnosis as follows:

$\sim$ "Cellulitis or abscess of the finger or toe" (ICD-9-CM 681.xx)

"Other cellulitis or abscess" (ICD-9-CM 682.x)

"Other local infection of the skin or subcutaneous tissue" (ICD-9-CM 686.xx)

"Posttraumatic wound infection not elsewhere classified" (ICD-9-CM 958.3)

"Other post-operative infection" (ICD-9-CM 998.59)

OR

- a diagnosis-related group (DRG) as follows:

"Cellulitis, age $>17$ with complications" (DRG 277)

"Cellulitis, age >17 without complications" (DRG 278)

"Post-operative or post-traumatic infection" (DRG 418)

\section{Exclusion criteria:}

- Hospitalized patients aged $<17$ years

- Hospitalizations with a length of stay $<1$ day

\section{Definition of a "non-severe" case:}

All selected admissions with a length of stay $\leq 3$ days

AND a diagnosis-related group (DRG) not including the following:

- "Subtotal mastectomy for malignancy with CC" (DRG 259)

- "Extensive procedure unrelated to the principal diagnosis" (DRG 468)

- "Ungroupable" (DRG 470)

- "Prostatic procedure unrelated to the principal diagnosis" (DRG 476)

- "Non-extensive procedure unrelated to the principal diagnosis" (DRG 477)

- "Tracheostomy for face, mouth and neck diagnoses" (DRG 482) 
- "Tracheostomy except for face, mouth and neck diagnoses" (DRG 483)

- "Extracorporeal membrane oxygenation or tracheostomy with mechanical ventilation $\geq 96$ hours or a principal diagnosis unrelated to the face, mouth and neck, with a major procedure" (DRG 541)

- "Tracheostomy with mechanical ventilation $\geq 96$ hours or a principal diagnosis unrelated to the face, mouth and neck, without a major procedure" (DRG 542)

\section{Algorithm to identify ABSSSI patients}

For mapping between ICD9-ICD10 codes, the "ICD-9 to ICD-10 Code Search IICD-10 Code Lookup \& Crosswalk" tool was used, and the correspondences were double-checked.

\section{Algorithm to identify ABSSSI patients in Spain}

The

Spanish

official

database

(http://pestadistico.inteligenciadegestion.msssi.es/publicosns) was searched for -CIE-9 681, 682 and/or DRG APR 383 codes.

\section{Algorithm to identify ABSSSI patients in Austria.}

In Austria, ABSSSIs are not as easily identified as in other countries. For example, many ABSSSIs, such as erysipelas, are coded as HDG19.07 "Komplizierte Affektionen der Haut", a DRG group that also includes pemphigus vulgaris. A sub-group (HG156) is available but still includes diseases such as ulcus cruris and decubitus. We therefore requested all records for the ICD 10 codes from the Italian algorithm (using an ICD-9 to ICD-10 Code Search IICD-10 Code Lookup \& Crosswalk) together with their DRG codes from the Austrian Ministry of Health. Then, we identified plausible ICD/DRG combinations with the following algorithm:

The DRG points of all acute-care stays of persons aged 20 years and over (the lowest category would have been 15-19) for the years 2008-20014 and the following:

$\sim \operatorname{LOS} 0,1,2-3,4+$ (for each category)

$\underline{\text { AND }}$

$\sim$ An ICD10 equivalent of Italian algorithm ICD9 codes

$\underline{\text { AND }}$

$$
\begin{aligned}
& \sim \text { HDG } 19.07 \\
& \sim \text { HDG } 19.08 \\
& \sim \text { HDG } 4.01
\end{aligned}
$$


$\sim$ MEL 06.05

MEL 09.03

〜 MEL 31.01

$\sim$ GERIAT

$\sim$ REMOB

$\sim$ LANGZEIT

$\sim$ PALLIAT

For each ICD10/DRG/LOS category, readmissions were also made available. Zero-day stays were not included in the cases but were requested for completeness. 